\title{
Development of High-Speed Ultrawideband Ground-Penetrating Radar for Rebar Detection
}

\author{
Xianlei $\mathrm{Xu}^{1}$; Tian $\mathrm{Xia}^{2}$; Anbu Venkatachalam ${ }^{3}$; and Dryver Huston, M.ASCE ${ }^{4}$
}

\begin{abstract}
This paper describes the development of new air-coupled ultrawideband ground-penetrating radar (GPR) for highway pavement and bridge deck inspections that can achieve high spatial resolution and high inspection performance while operating on vehicles driving at regular highway speeds. The major design features include dual-frequency band operation, 8 gigasamples per second high-speed real-time data acquisition, high-speed large volume data transmission and storage, and customized signal-processing algorithms for GPR image enhancement and feature extractions. For design validations, steel reinforcing bar detection experiments in various setups are conducted. The measurement results show good agreement with the subsurface feature configurations. DOI: 10.1061/(ASCE)EM.1943-7889.0000458. @ 2013 American Society of Civil Engineers.
\end{abstract}

CE Database subject headings: Structure reinforcement; Sampling; Imaging techniques; Radar.

Author keywords: GPR system; Rebar detection; High-speed real-time sampling; Air coupled; Imaging processing.

\section{Introduction}

The development of noninvasive techniques to identify and classify subsurface features has generated interest in a wide variety of application fields, such as oil and gas exploration, geology, conduit and pipe localization, and archeology (Pasolli et al. 2009; Chang et al. 2009). To this end, the research reported here is in the development of ground-penetrating radar (GPR) for identifying the location and condition of steel reinforcing bars in concrete bridge decks (Roqueta et al. 2012; Dérobert et al. 2008; Shaw et al. 2003; Halabe et al. 1995). Such noninvasive techniques provide useful information for maintenance and condition assessment efforts. Of particular interest is using GPR in highway or bridge deck subsurface inspections without disrupting vehicular traffic.

A variety of GPR systems are currently available. Based on antenna installations, GPR can be classified as ground-coupled GPR or air-coupled GPR. The ground-coupled GPR has its antennas installed at close proximity to the detection surface, which results in high detection sensitivity and small signal loss. However, when installed at this height, antennas are prone to damage if the surface under detection has a rough profile. Consequently, the ground-coupled GPR survey speed is low, and its operation may cause traffic disturbances. In air-coupled GPR, the antennas are typically installed $200-500 \mathrm{~mm}$ or even higher above the surface. Hence, GPR survey speed can be increased without concerns of

\footnotetext{
${ }^{1}$ Visiting Graduate Student, School of Engineering, Univ. of Vermont, Burlington, VT 05405. E-mail: xxu1@uvm.edu

${ }^{2}$ Associate Professor, School of Engineering, Univ. of Vermont, Burlington, VT 05405. E-mail: txia@uvm.edu

${ }^{3}$ Graduate Student, School of Engineering, Univ. of Vermont, Burlington, VT 05405. E-mail: Anbu.Venkatachalam@uvm.edu

${ }^{4}$ Professor, School of Engineering, Univ. of Vermont, Burlington, VT 05405 (corresponding author). E-mail: dryver.huston@uvm.edu

Note. This manuscript was submitted on November 1,2011; approved on July 9, 2012; published online on July 30, 2012. Discussion period open until August 1, 2013; separate discussions must be submitted for individual papers. This paper is part of the Journal of Engineering Mechanics, Vol. 139, No. 3, March 1, 2013. @ASCE, ISSN 0733-9399/ 2013/3-272-285/\$25.00.
}

damage to the antennas. There are some commercial air-coupled GPRs that can operate at highway speed (e.g., $96.5 \mathrm{~km} / \mathrm{h}$ ). However, the data acquisition units in most of these systems are designed using the low rate equivalent-time sampling method. As a result, the effective road inspection speed is low. Uddin (2006) reported that most in commercial highway speeds (i.e., $96.5 \mathrm{~km} / \mathrm{h}$ posted) air-coupled GPRs transmit and receive 50 pulses per second. When the GPR vehicle drives at $96.5 \mathrm{~km} / \mathrm{h}$, a low pulse transmission rate will lead to low spatial resolution in the inspection, which results in the production of blind spots that miss many subsurface features in the characterization.

In the United States, Federal Communications Commission (FCC) regulations severely limit ultrawideband (UWB) radiated emissions in the frequency band from 0.96 to $3.1 \mathrm{GHz}$, which happens to be favorable in GPR roadway inspections (FCC 2002). This constraint and the aforementioned limiting factors are challenges in GPR designs. This research presents efforts to overcome these constraints and limitations by developing a new and small airlaunched UWB GPR system that can be installed at the bottom side of a vehicle. A key feature is the use of a high-speed real-time sampling unit that acquires the full reflected GPR waveforms in a single shot, which bypasses the slower subsampling methods used by most presently available impulse radar systems. By configuring the data acquisition unit in a multithread operating mode, an enormous amount of sampled data can be rapidly collected and stored. These improvements collectively enable this new UWB radar to operate in a vehicle running at normal driving speed.

Another challenge in GPR design is that various forms of interference and noise couplings can corrupt GPR data and deteriorate system performance. In this research, a series of performanceenhancing signal-processing approaches are developed, including data preprocessing for systematic noise and interference reduction, target area detection, and linear hyperbola fitting to automate GPR data characterization and to leverage radar operating efficiency and accuracy. For design validation, experiments are conducted by means of various test setups.

The organization of this paper is as follows. A short description of the GPR system hardware and data acquisition unit designs is presented. Then, the signal preprocessing algorithms developed to 
improve rebar detection and signature characterizations are given. Experimental results are presented and, finally, concluding remarks are given.

\section{GPR Hardware Development}

\section{System Issues}

The UWB GPR is an impulse radar system in which a short electromagnetic (EM) pulse launched from an antenna travels through air and ground dielectric media to interact with subsurface features. A receiver collects the reflected and scattered impulses for data storage, analysis, and subsurface feature identification. The radar hardware consists of a radio-frequency (RF) front end and digital control and data processing back end, including the data acquisition module. The UWB pulse generator is a major front-end component that plays a critical role in determining the GPR system bandwidth, the detection range resolution, and the EM wave penetration capability. The data acquisition unit is a crucial back-end component. Its sampling speed directly determines whether the GPR can operate at regular highway driving speeds while achieving sufficient spatial resolution. In most GPR systems (Jol 2009; Gan et al. 2011), equivalent-time subsampling provides a practical and low-cost method of digitizing the high-speed waveforms. Equivalent-time subsampling is based on the assumption that the object under test is static or moves slowly, relative to GPR transceiver. Thus, the reflection signal within multiple sampling cycles is periodic and has an invariant waveform. By sampling various portions of the reflection signal across multiple cycles, the reflection waveform of a complete cycle can be reconstructed over time. However, for a highway speed GPR system, the foundation for equivalent-time sampling may lose its validity. When the GPR vehicle travels at a high speed (e.g., $30 \mathrm{~m} / \mathrm{s}$ ), the GPR transceiver can move with a relatively large spatial offset during the waveform reconstruction intervals. Consequently, the reflection signal will not be periodic and may become unsuitable for equivalent-time sampling. To reduce the spatial offset during the wave reconstruction, the radar pulse has to be launched more frequently. However, increasing the pulse launching frequency will increase the radiated emission power that is constrained by FCC regulation. The new GPR reported here exploits high-speed real-time sampling techniques to realize single-shot data collection, where the reflection signal in a complete cycle is obtained in a single trigger event with high fidelity.

Another advantage of applying real-time sampling is that it can help improve highway GPR survey resolution. For detecting a buried rebar at normal highway speed, the relative target moving speed and the data acquisition speed are related as

$$
\Delta t=\frac{\delta R}{v}=\frac{1 \mathrm{~cm}}{96.5 \mathrm{~km} / \mathrm{h}}=0.37 \mathrm{~ms}
$$

where $\delta R=$ targeted image cell resolution of $1 \mathrm{~cm} ; \nu=$ relative target moving speed (which equals the normal highway driving speed of $\sim 96.5 \mathrm{~km} / \mathrm{h}$ ); and $\Delta t=$ allowed data acquisition time for sampling reflection signals within two consecutive cycles. The $\Delta t$ value determines the applicable minimum GPR pulse repetition frequency (PRF), which equals $2.7 \mathrm{kHz}(=1 / 0.37 \mathrm{~ms})$ when the GPR vehicle moves at $96.5 \mathrm{~km} / \mathrm{h}$. In fact, for practical GPR operations, the PRF typically needs to be 10 times higher than the minimum PRF, which will enable sampling the same location reflection signal multiple times to facilitate data averaging for noise alleviation. If the equivalent-time sampling method is adopted, then it would require an even higher PRF for multicycle waveform sampling and signal reconstruction. A higher PRF produces more emission pulses within a shorter time interval, which leads to a higher level of time-averaged radiated emission power density. In order for UWB radar to meet the emission power constraints regulated by FCC 02-48 (FCC 2002), the GPR pulse amplitude has to be reduced as a design trade-off, which will degrade radar system performance and its detection efficiency. These factors reinforce the suitability of real-time full-waveform sampling for a highway speed GPR system.

\section{System Configuration}

Typically, for radar signals low-frequency EM waves have good penetrating capability while high-frequency EM waves can achieve better spatial resolution. To accomplish high detection resolution and good penetration while reducing power radiation in the $0.93-$ 3.1 GHz band, a dual-band dual-channel UWB GPR has been developed. Fig. 1 illustrates the system diagram. In the design, the UWB pulse generator receives an oscillating signal from a programmable oscillator whose frequency is tunable from $1 \mathrm{kHz}$ to $10 \mathrm{MHz}$ to control the UWB PRF. The UWB pulse generator generates 600-ps-wide monocycle pulses as the source signal for both sub-1-GHz base and 3-4.5-GHz high bands. In the base-band operation, the monocycle pulse is directly amplified, emitted, and received through UWB antennas, while at the high-frequency band the monocycle pulse feeds into a mixer circuit (Hittite HMC128G8) to perform frequency modulation by a $3-\mathrm{GHz}$ local oscillator. The spectrum of the resulting emitted and received signal spans from 3 to about 4.5 GHz. Two remote-controlled single-pole/dual-throw RF switches are used to select the operating band. A field programmable gate array (FPGA) circuit (Xilinx Spartan-3) provides the high-speed control. To improve radar survey efficiency, the two operating channels are implemented to realize parallel inspections, with each channel having its own transmission antenna and receiving antenna. To minimize interchannel coupling and interference, a Ping-Pong mode control scheme turns on each channel alternately. At the receiver side, various band signals are processed differently. The base-band signal is amplified and filtered before analog-to-digital (ADC) conversion. The high-band signal uses a mixer to perform frequency down conversion using the same local oscillator as that in the transmitter. The FPGA coordinates the radar operating band selection, channel switching, sampling data collection, and storage.

\section{Pulse Generator}

In GPR radar design, it is important to develop a UWB pulse generator that can produce high-quality pulses with large-amplitude, low-level ringing and a wide range of applicable PRFs. This research develops a high-quality and low-cost pulse generator circuit using a step recovery diode (SRD) and short-circuited microstrip lines. As shown in Fig. 2(a), the pulse generator consists of the following three functional units: (1) a signal amplitude converter, (2) a Gaussian pulse generator, and (3) a pulse-shaping filter. The signal amplitude converter contains a current feedback operational amplifier (THS3091), a negative voltage converter (TL 7660), and a 50$\Omega$ terminal resistor. The main function is to convert a single polarity square wave $\left(0 \mathrm{~V}\right.$ to $\left.\mathrm{V}_{\mathrm{dd}}\right)$ to a dual-polarity square wave $\left(-\mathrm{V}_{\mathrm{dd}}\right.$ to $\mathrm{V}_{\mathrm{dd}}$ ). The dual polarity signal $V_{s}$ acts as the stimulus signal for the SRD Gaussian pulse generator unit. Inserting a power attenuator alleviates the SRD nonlinearity caused by impedance matching. The SRD sharpens the input square-wave transition edge by connecting to the short-circuited microstrip lines through PIN diodes and directcurrent blocking capacitors. Switching a specific microstrip line turns on a forward-biased PIN diode to connect the edge-sharpened 

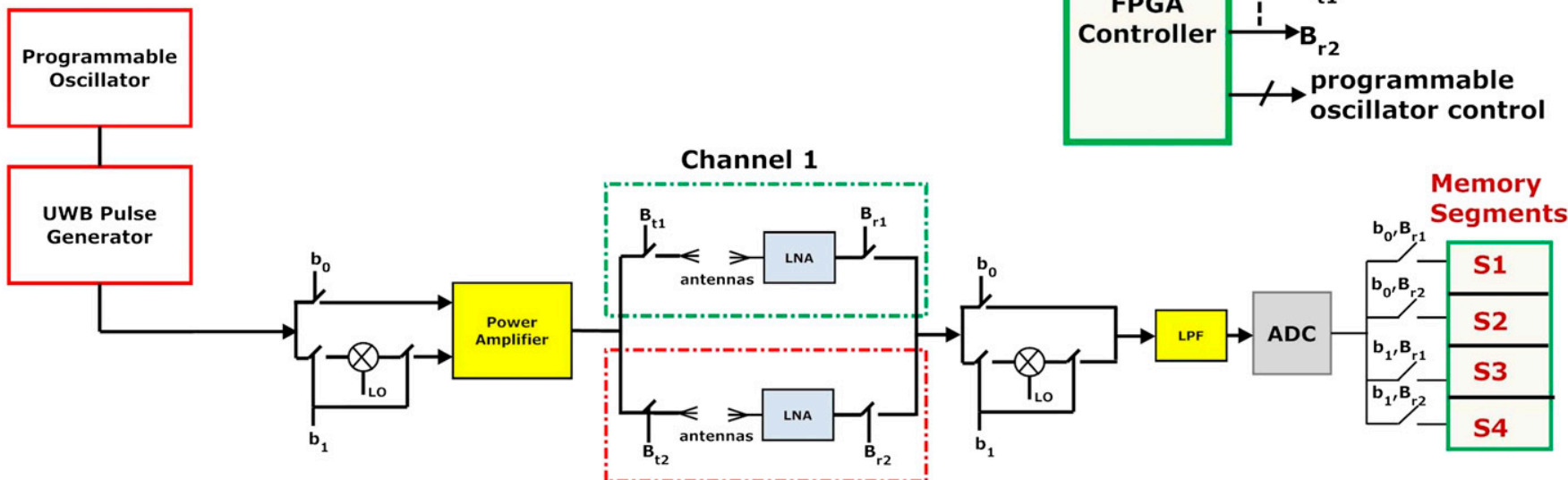

Channel 2
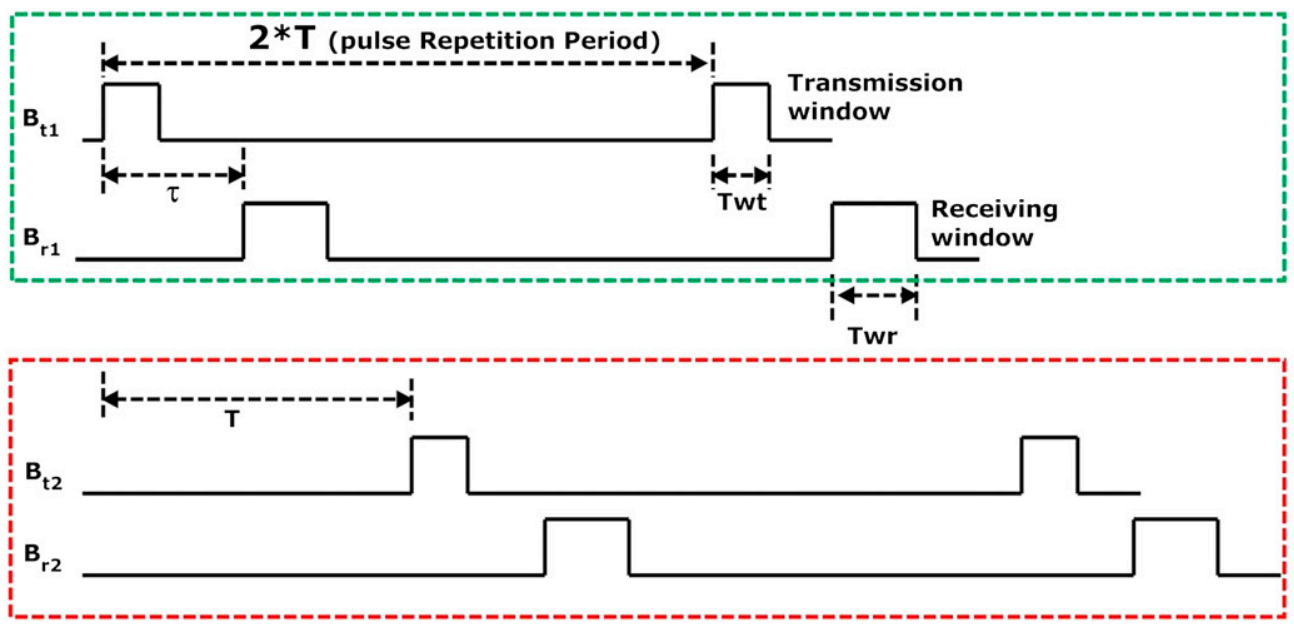

Fig. 1. Block diagram of the high-speed UWB dual-band GPR system

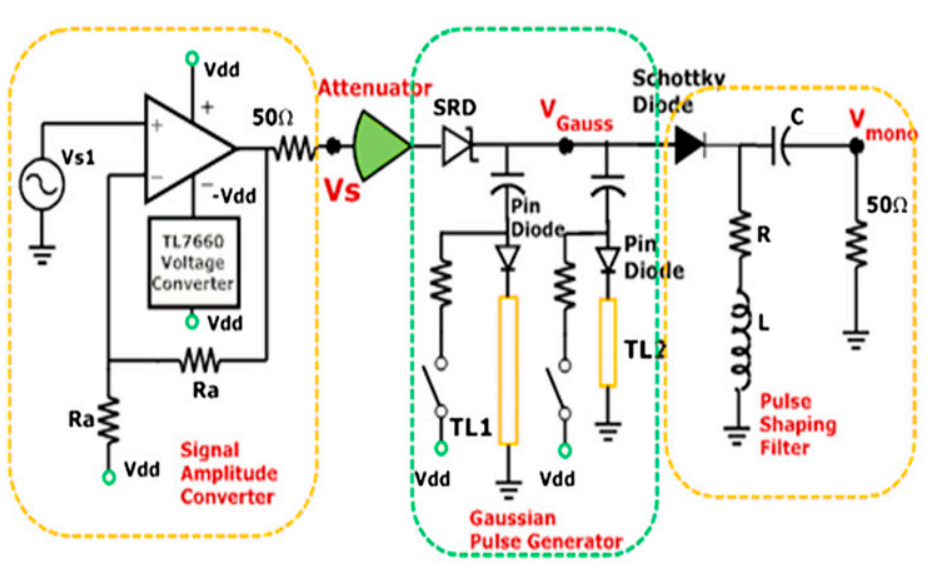

(a)

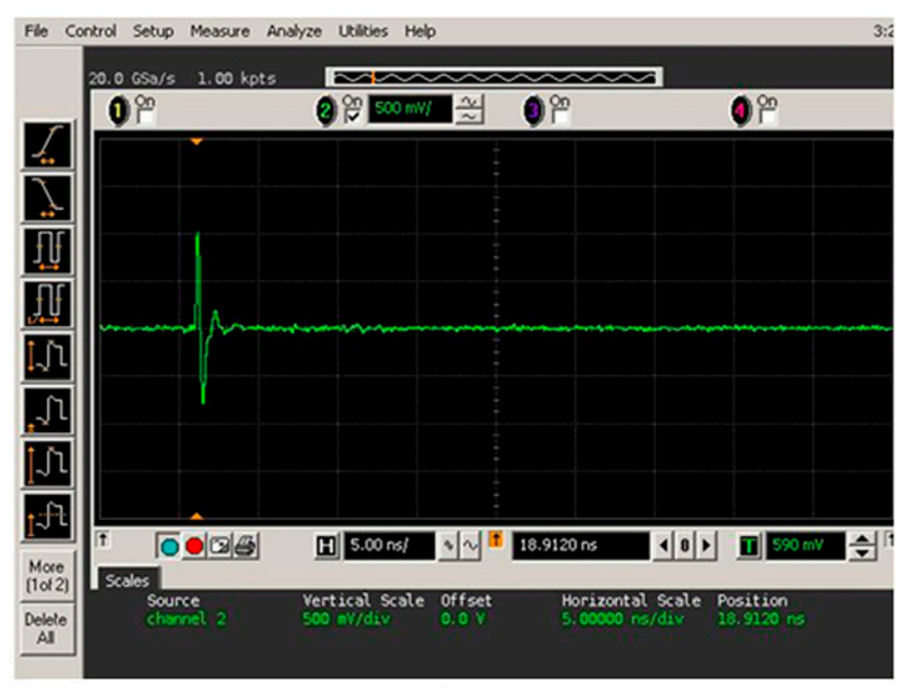

(b)

Fig. 2. (a) UWB monocycle pulse generator circuit; (b) pulse measurement result 
step signal to the microstrip line. The short-circuit nature of the microstrip causes the step signal traveling along the microstrip line to be fully reflected with an opposite phase. At the SRD output, the incident step signal and the reflection signal add together to produce a Gaussian pulse, in which the width is determined by the signal propagation delay along the microstrip line and is proportional to the microstrip line length. Using PIN diodes to select microstrip lines of various lengths enables tuning the Gaussian pulse width. The Gaussian pulse then connects to a Schottky diode, which acts as a half-wave rectifier to pass only the positive pulses while eliminating the negative ones. The pulse shaping unit consists of a shunt resistor, an inductor, and a series-connected capacitor. The resistor and inductor have small values to produce a low-impedance path to shunt the low-frequency ripples to the ground, while the small capacitor in the series passes only the high-frequency components in the Gaussian pulse. This pulse-shaping circuit plays two roles (Xia et al. 2012). On one hand, it acts as a high-pass filter to eliminate

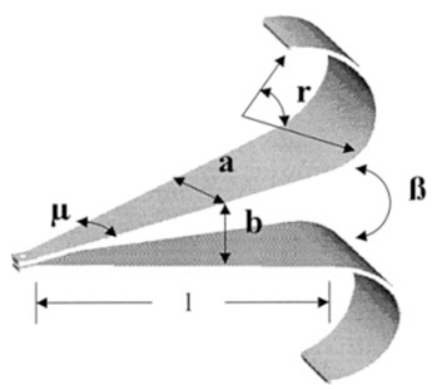

Fig. 3. Drawing of UWB horn antenna, where $\mu=13^{\circ}, \beta=6^{\circ}$, $r=150^{\circ}, a=60 \mathrm{~mm}, b=60 \mathrm{~mm}$, and $l=180 \mathrm{~mm}$ (reprinted from Journal of Applied Physics, 43, Huston, D. R., Hu, J. Q., Maser, K, Weedon, W, Adam, C. GIMA Ground Penetrating Radar System For Infrastructure Health Monitoring, 139-146, Copyright 2000; with permission from Elsevier)

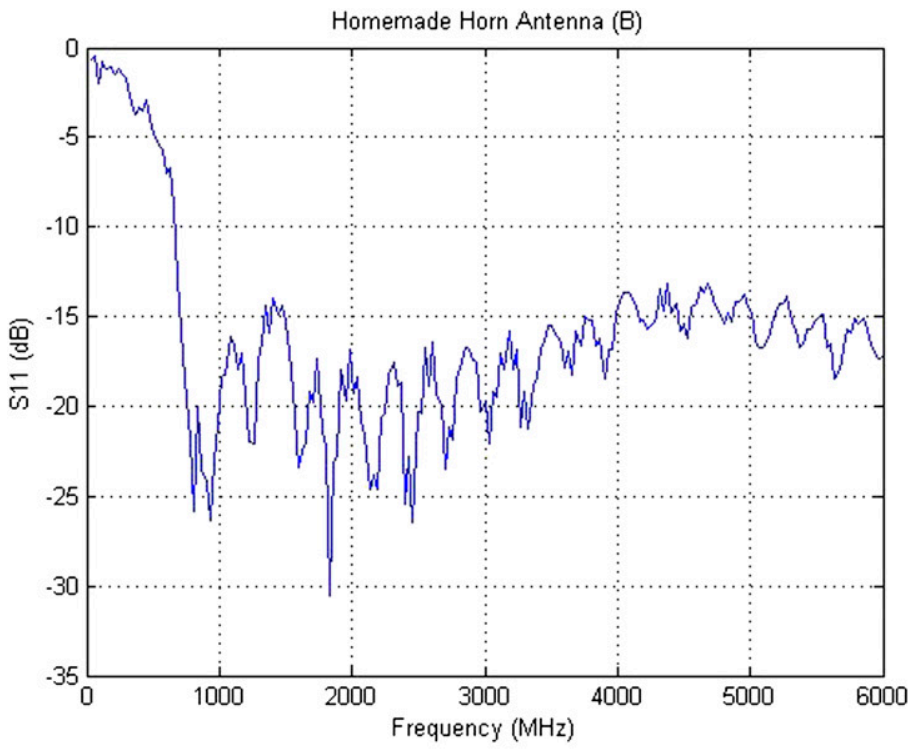

(a) low-frequency ripples; on the other hand, it acts as a differentiator to produce a monocycle pulse from the input Gaussian pulse. The differentiator causes the monocycle pulse amplitude to be proportional to the slope of the Gaussian pulse. Because the Gaussian pulse is very narrow (less than $1 \mathrm{~ns}$ in this design) and has sharp transition edges, the resulting monocycle pulse amplitude is high. Fig. 2(b) shows the measurement of a Gaussian monocycle pulse, which shows that the pulse width is approximately $600 \mathrm{ps}$ and the amplitude is approximately $1.8 \mathrm{~V}$. When compared with other designs in the literature, this pulse generator achieves lower levels of ringing and higher pulse amplitudes.

\section{Ultrawideband Antenna}

To have the maximum power transmitted onto the object under inspection, the GPR antennas need to have good impedance matching to minimize the internal reflections and to smooth out the transition from the circuit impedance, $50 \Omega$, to the free space impedance, $377 \Omega$. In this study, the GPR system is a novel homemade good impedance match antenna (GIMA) (Huston et al. 2000). As shown in Fig. 3, this GIMA antenna appears as a waveguide with two metal conductors of varying widths that are separated by an insulator. Fig. 3 illustrates the shape and the critical dimensions of the GIMA antenna.

The design concept of the GIMA is to provide a smooth impedance transition from the antenna to the air. This is accomplished with a combination of taper and flare at the ends of the antenna horns. The variation in impedance is achieved by smooth variation of the width (angle $\alpha$ ) of metal plates and the separation gap $(\beta)$ between the metal plates with rolled edges to minimize edge diffraction, and an impedance-matched integrated-balun feed-point connection. The immediate free space surrounding the antenna conductors are covered with energy absorbing material to minimize energy dissipation and to improve signal directivity. The developed GIMR antenna has steady impedance in the operational band from $500 \mathrm{MHz}$ to $6 \mathrm{GHz}$. Fig. 4(a) plots the measurement results of Return Loss S11, whereas Fig. 4(b) illustrates the voltage standing-wave ratio (VSWR).

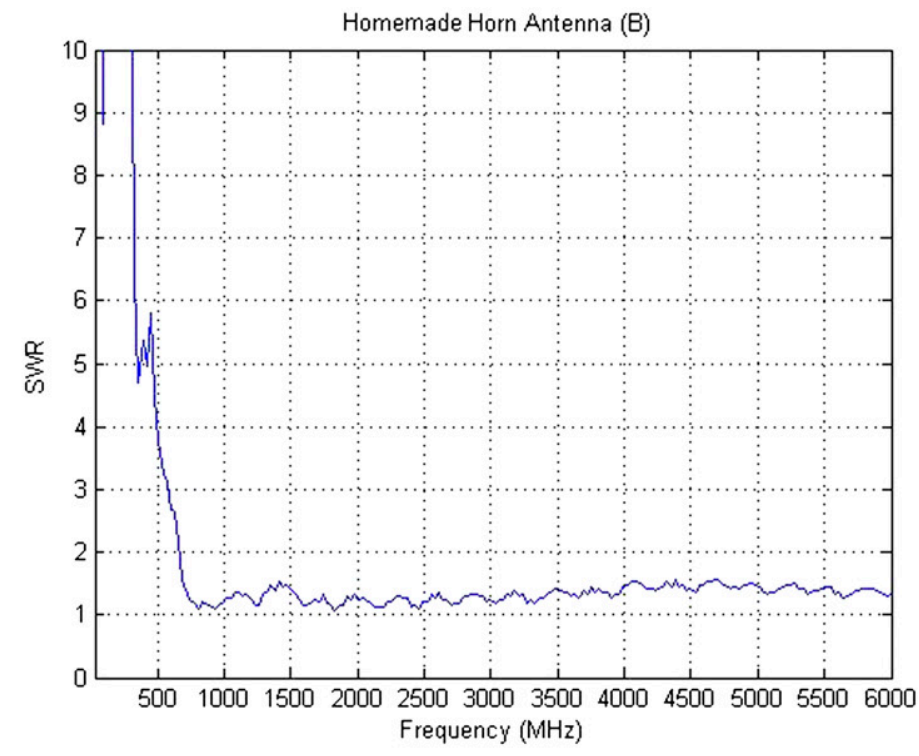

(b)

Fig. 4. (a) Reflection loss of the UWB horn antenna from $30 \mathrm{MHz}$ to $6 \mathrm{GHz}$; (b) signal wave ratio of the UWB horn antenna from $30 \mathrm{MHz}$ to $6 \mathrm{GHz}$ 
It is clearly seen that the GIMA return loss is less than $-10 \mathrm{~dB}$ and the VSWR is less than 1.4 across the frequency band. These results attest to the good impedance-matching performance of this antenna design.

\section{High-Speed Digitizer Configuration}

The digitizer used in this system is a real-time ADC converter (Agilent Acqiris U1065A) of 8-gigasamples per second (GSPS) sampling rate and 10-bit resolution. This high-speed and high-resolution digitizer enables implementing a single-shot full-waveform UWB scanning system for highway bridge inspection at normal driving speed. During operation, a large amount of data is collected at the rate of 8 GSPS. The rapidly accumulated large volume of data is difficult to manage with ordinary read and write operations. The design solution is to configure the digitizer into the multibuffer simultaneous acquisition and readout (SAR) mode. In the SAR mode, the internal memory of the digitizer is divided into three banks, as shown in Fig. 5, to form a circular structure. Each data bank supports dual-port data access to allow simultaneous data writing and reading. In other words, the stored data can be read out from one memory bank while the new incoming acquisition data can be written into another available free bank. A further configuration is to operate the data acquisition bank in sequence acquisition mode, in which the bank is divided into 333 segments. Each segment has the capacity to store 320 data samples sequentially.

Upon triggering, the digitizer starts one data acquisition process, named a trigger event. At the end of each trigger event, there is

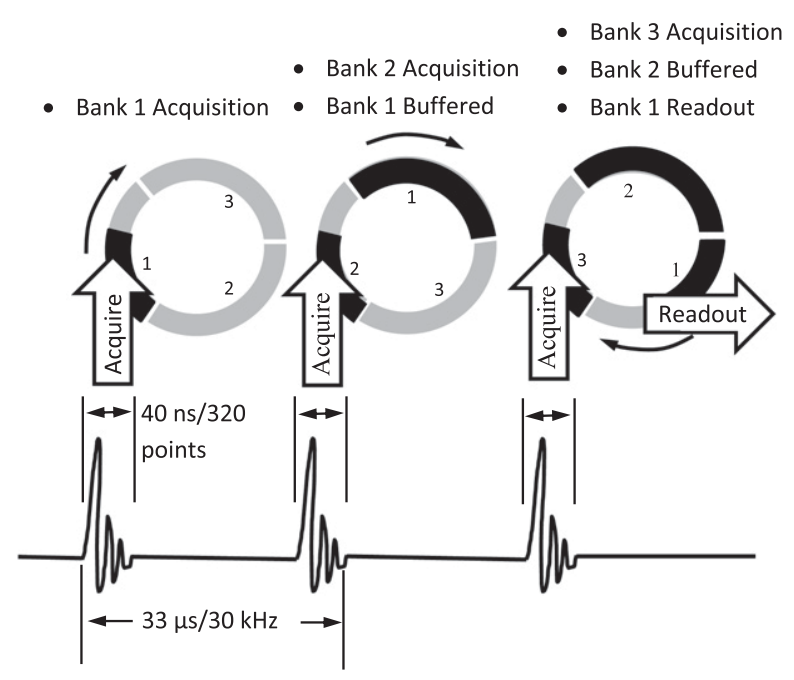

Fig. 5. Digitizer configured in the SAR mode a short time interval in which the digitizer stops new data acquisition. This time interval is the rearm time or dead time, which affects the maximum event rate or trigger frequency that the digitizer can operate without missing recording. One valuable feature of the sequence acquisition mode is that it reduces the rearm time or dead time to less than $350 \mathrm{~ns}$ at the 8-GSPS sampling rate.

The digitizer operations take on average approximately $13 \mathrm{~ns}$ to transfer each data sample from the internal memory of the digitizer to the back-end computer hard disk. However, acquiring each data sample only takes $125 \mathrm{ps}$. The apparent data transfer speed is about 100 times slower than the sampling data acquisition speed. Such a large speed discrepancy could cause digitizer operation jam and data losses. Fortunately, the impulse GPR signal has an extremely low duty cycle and the significant time interval is only a small portion of the whole signal period. Fig. 5 shows that for a 30-kHz PRF (33.3$\mu$ s period), the effective sampling window is only 40-ns wide and only 320 sampling data points are collected. To fill up one memory bank, it takes about $1 \mathrm{~ms}(33.3 \mu \mathrm{s} /$ segment $\times 333$ segments $=$ $1 \mathrm{~ms}$ ), while the effective sampling time length is only $13.32 \mu \mathrm{s}$ $(40 \mathrm{~ns} /$ segment $\times 333$ segments $=13.32 \mu \mathrm{s})$. This gives nearly a 1-ms time margin for transferring the data collected from the digitizer to the computer hard disk. In other words, this buffered pipelining scheme compensates for the speed mismatch by a factor of $76(=1 \mathrm{~ms} / 13.32 \mu \mathrm{s})$.

To further alleviate the speed mismatch, as shown in Fig. 6, a multiprocessor configuration was also implemented to realize multithread data reading and writing in order to speed up data migration onto the computer hard disk, where one processor reads data from the digitizer while three processors move data to the hard disk in parallel. Use of these techniques effectively resolves the speed mismatch between GPR signal sampling and data storage.

\section{Rebar Test Development}

The GPR is designed for rebar detection and feature characterization. A focus of this paper is on locating the buried rebar. To illustrate the design effectiveness, a series of experimental studies were conducted with three different rebar test configurations: (1) one 20$\mathrm{mm}$-diameter rebar was positioned in air $450 \mathrm{~mm}$ below the antennas and $550 \mathrm{~mm}$ above the floor, (2) one 15-mm-diameter rebar was buried $100 \mathrm{~mm}$ deep inside a sandbox (the sandbox top surface was $250 \mathrm{~mm}$ below the antennas), and (3) a concrete slab with two 20-mm-thick rebars was embedded at depths of 108 and $98.6 \mathrm{~mm}$, respectively. The distance between the two rebars was $500 \mathrm{~mm}$. The antennas were $100 \mathrm{~mm}$ above the top surface of the slab. Fig. 7 illustrates each test diagram, whereas Fig. 8 contains photographs of all test configurations.

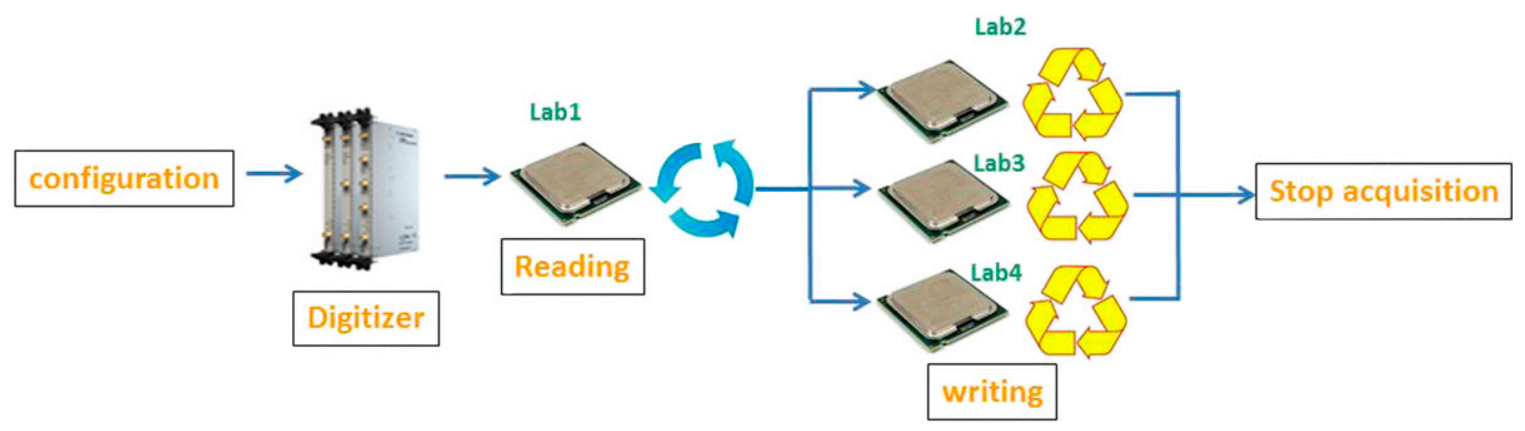

Fig. 6. Multithreading configuration for data transfer and storage 


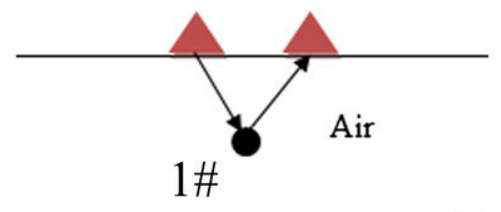

(a)

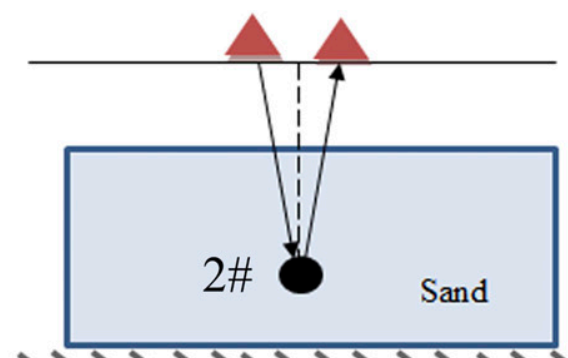

(b)

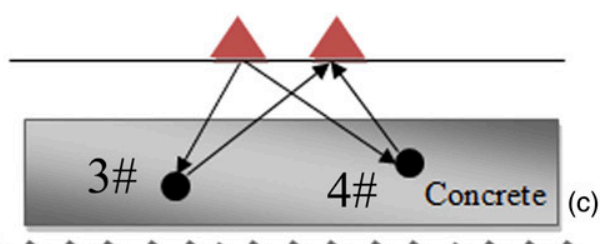

Fig. 7. Three different rebar test setups (triangles represent antennas; circular objects represent the rebars): (a) one rebar suspended in air; (b) one rebar buried in sand at a depth of $80 \mathrm{~mm}$; (c) two rebars in a concrete slab
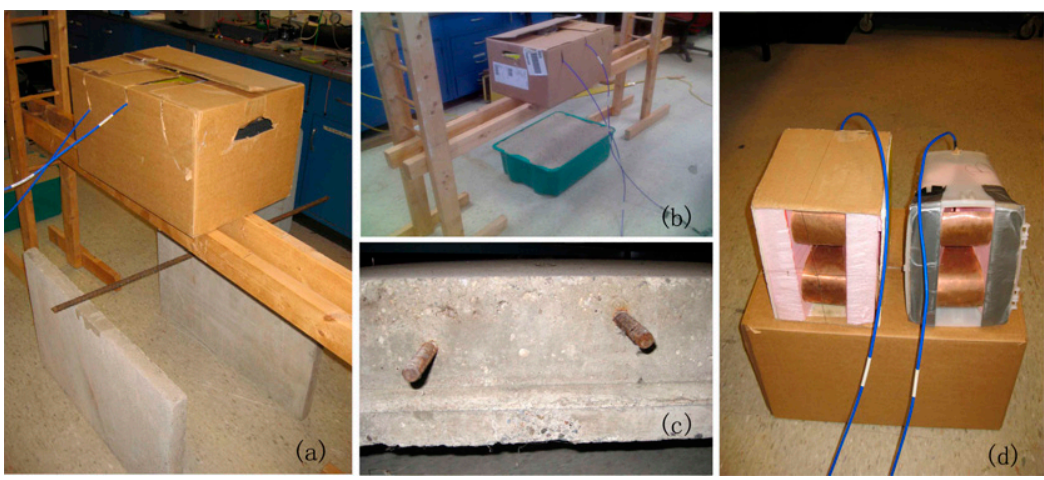

Fig. 8. Photographs of the rebar test setups (packed inside a hardboard box during the experiments): (a) rebar in air; (b) rebar in the sandbox; (c) two rebars in concrete slab; (d) two GIMA antennas
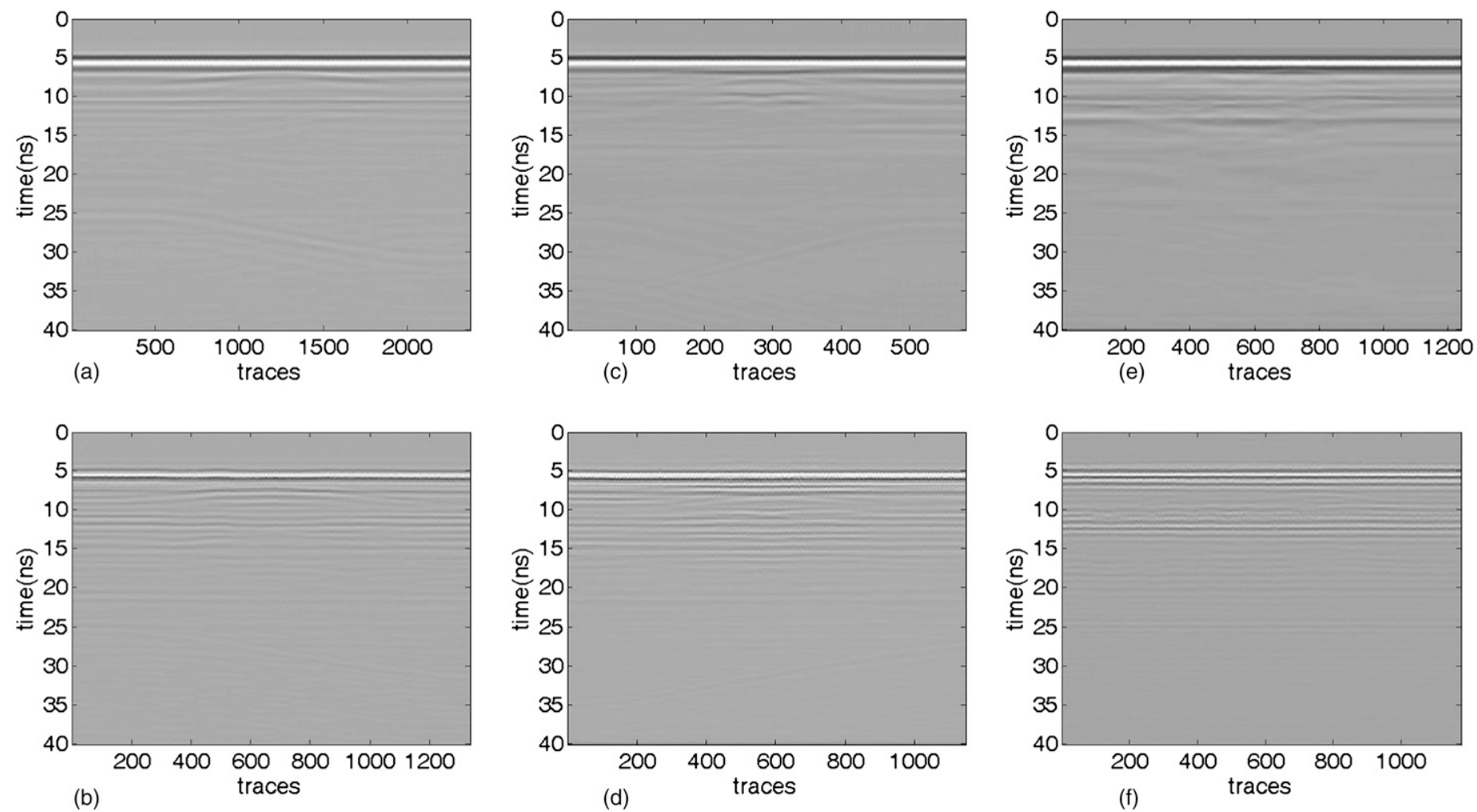

Fig. 9. Dual-band GPR B-scan images plotted from raw data without signal processing: (a) one rebar in air (base-band image); (b) one rebar in air (high-frequency band image); (c) one rebar in sand (base-band image); (d) one rebar in sand (high-frequency band image); (e) two rebars in concrete slab (base-band image); (f) two rebars in concrete slab (high-frequency band image) 

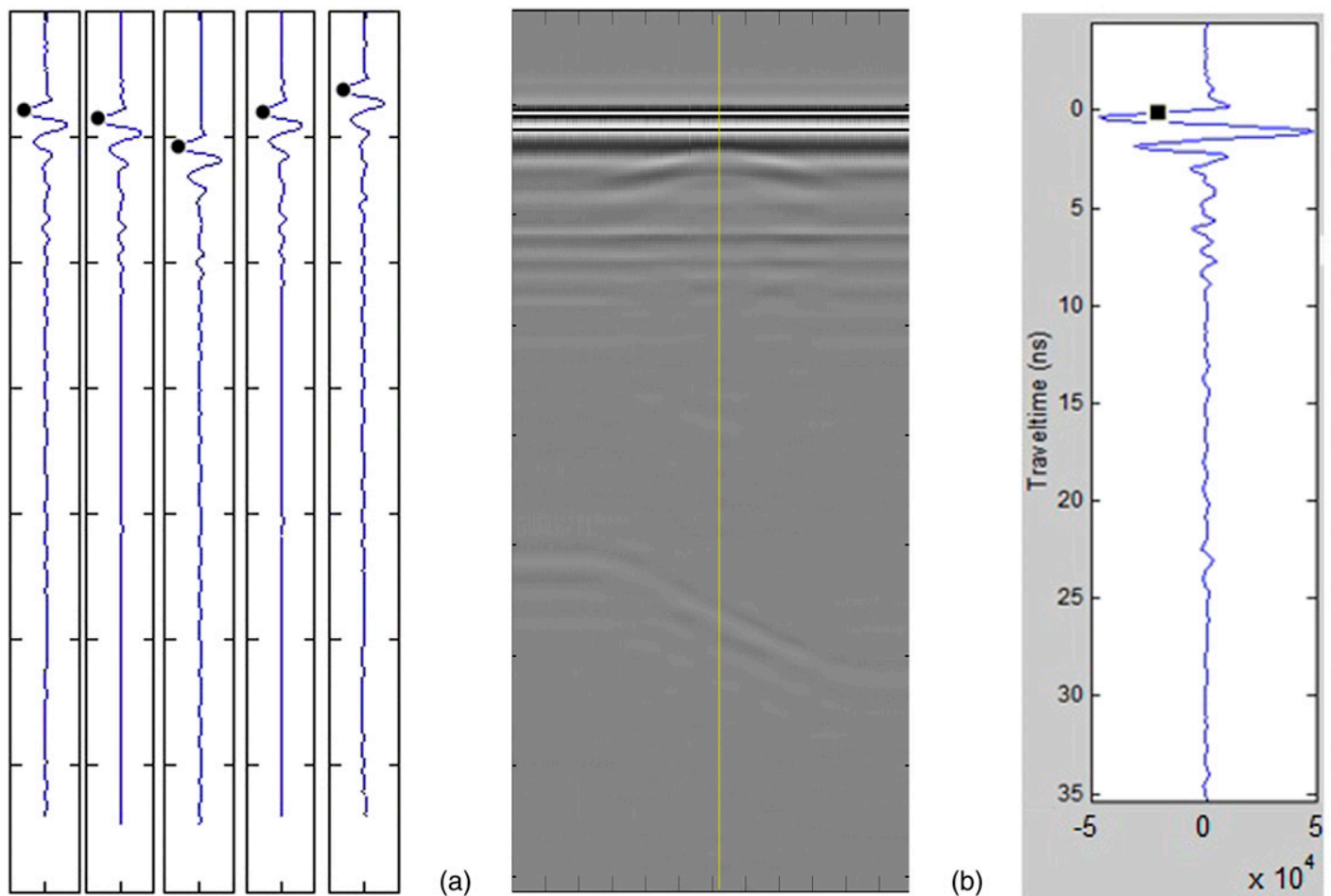

(c)

Fig. 10. (a) Waveforms of multiple traces showing level tracking; (b) image after vibration effect correction; (c) highlighted trace waveform showing time zero point selection
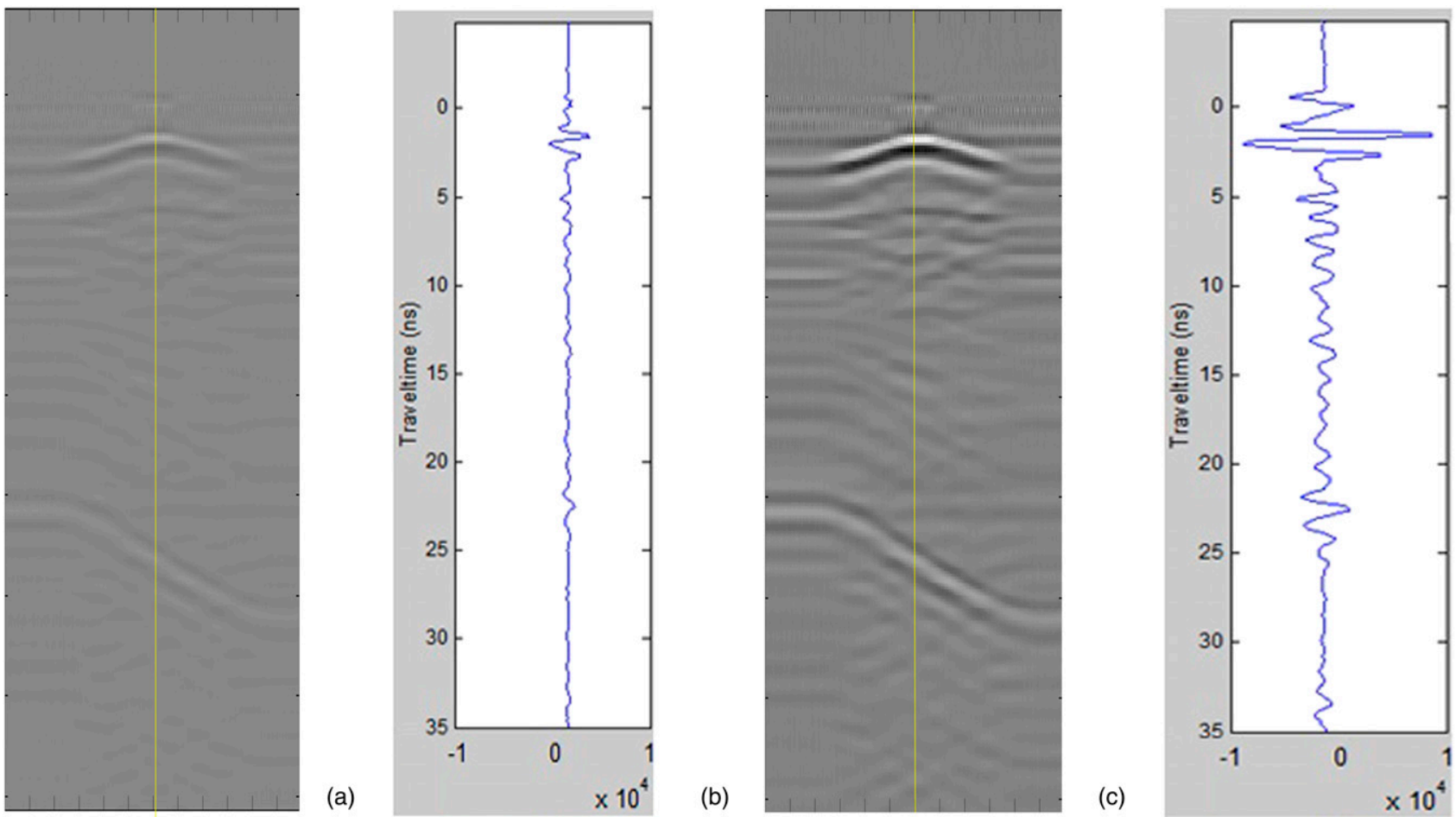

(d)

Fig. 11. (a) Image after systematic noise removal; (b) waveform corresponding to the highlighted trace in Fig. 10(a); (c) image of median filtering in RFI reduction; (d) waveform corresponding to the highlighted trace in Fig. 10(c) 


\section{Test Data Acquisition}

In the GPR scanning experiments, the antennas moved in a horizontal line above the rebar setup, while a series of reflection signals were sampled, recorded into computer data files, and stacked into a B-scan image (Fig. 9). As a result of large noise and interference, the rebar images were fuzzy and the signatures of individual rebars were difficult to recognize.

\section{Data Processing}

GPR image processing has been widely studied in the technical literature (Johansson and Mast 1994; Olhoeft 2000; Al-Qadi et al. 2004; Kim et al. 2004; Shihab and Al-Nuiamy 2005; Borgioli et al. 2008; Wang et al. 2011; Yang 2011). This section presents

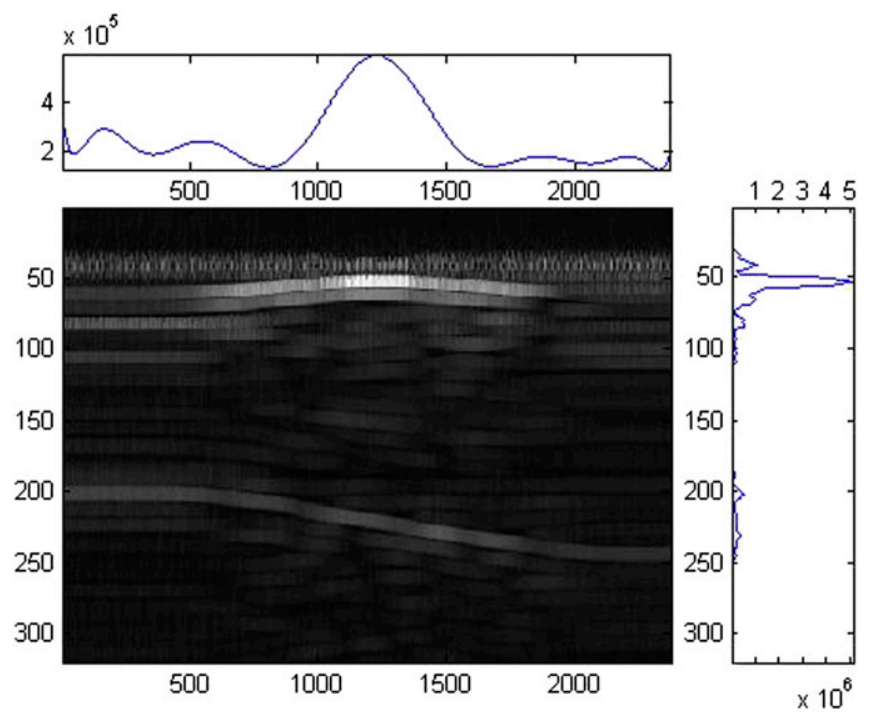

Fig. 12. GPR image upon morphological filtering the signal-processing algorithms that have been developed to improve rebar image quality and rebar detection accuracy. The proposed signal processing consists of three subcomponents, including data preprocessing, target area detection, and linear hyperbola fitting.

\section{Radar Data Preprocessing}

Data preprocessing removes undesired systematic noise and interference. It involves level tracking, level correction, time zero line setting, background clutter removal, and low-pass filtering. For the purpose of illustration, data from the rebar-in-air test configuration are used herein.

\section{Vibration Effect Correction}

In a scan test, mechanical vibrations in the vehicle suspension and antenna mounts cause relative movements of the antennas to the surface under inspection and can lead to spurious level misalignments among various sampling traces. For instance, in Fig. 10(a), the highlighted black dots on various traces are misaligned, although they are expected to be on the same horizontal line. Implementing level tracking and level correction algorithms can correct these waveform misalignments. For level tracking, a peak level is selected and identified on all waveform traces, along with their time indexes. Level correction selects the peak point index on the first trace as the reference, and adjusts the time indexes of all other traces by the relative offsets. This aligns the peak points on all traces on the same horizontal line with the same index value. Upon finishing level tracking and level correction, the time zero line is identified. In this project, the antenna installation plane is selected as the time zero reference plane. The installation configuration places the transmit and receive antennas in close proximity, which induces a strong EM coupling between the antennas. Thus, the first peak point on the received signal waveform is attributed to the direct coupling and can be recognized easily. As shown in Fig. 10(c), time zero is selected to be the midpoint on the transition edge because the transition slope has the largest value for easy identification.

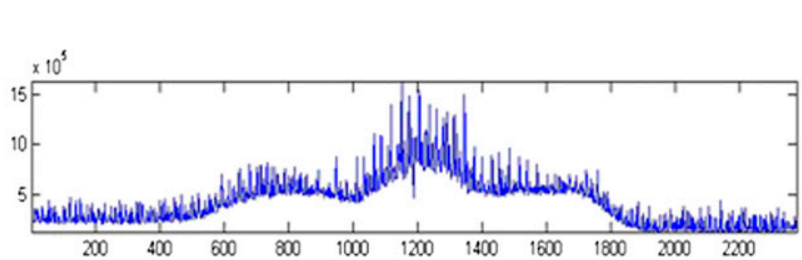

(a)

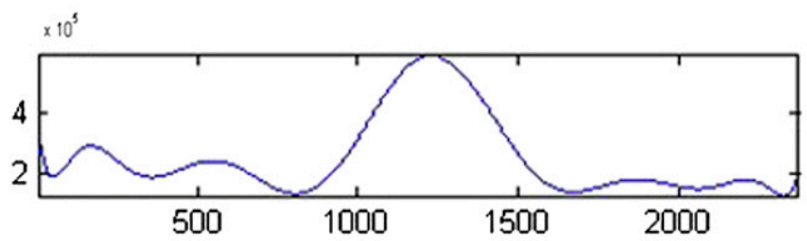

(b)
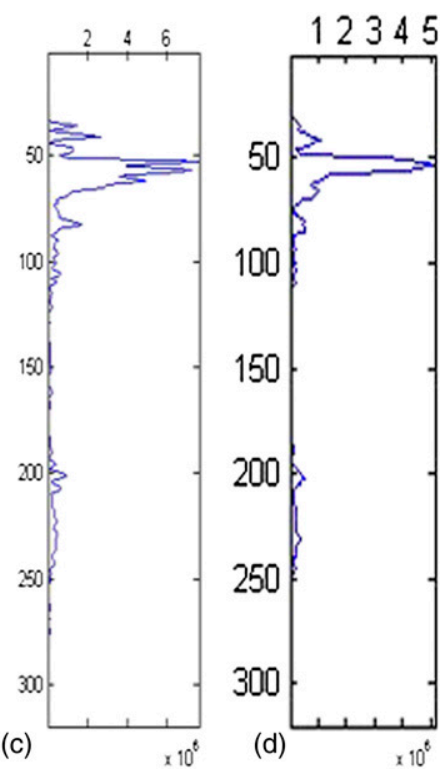

Fig. 13. Comparison of the normalized energy data before and after morphological filtering: (a) horizontal normalized energy map before filtering; (b) horizontal normalized energy upon filtering; (c) vertical normalized energy map before filtering; (d) vertical normalized energy upon filtering 

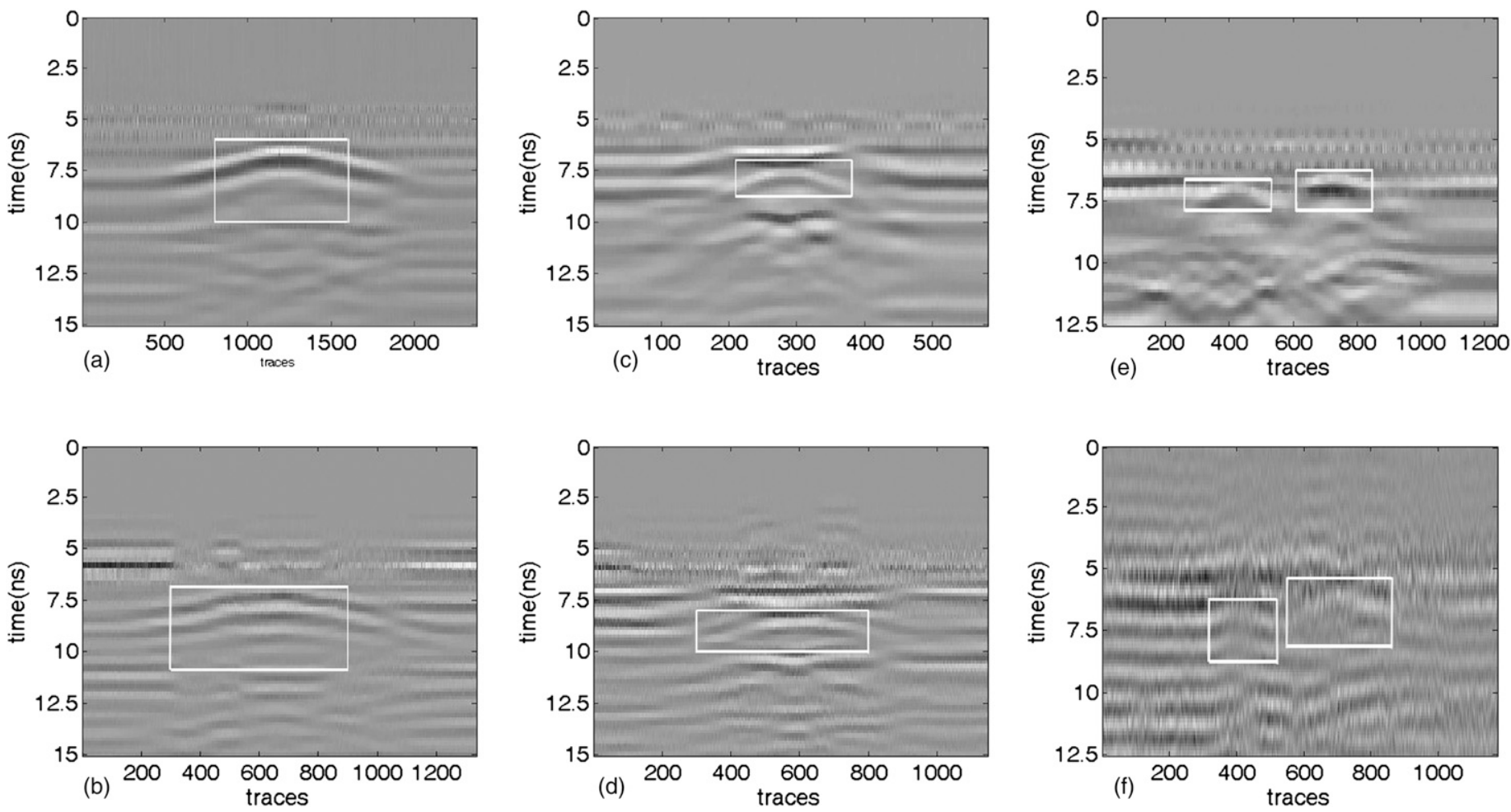

Fig. 14. Target area detection results for each test setup: (a) one rebar in air (base-band radar image); (b) one rebar in air (high-frequency band radar image); (c) one rebar in sand (base-band radar image); (d) one rebar in sand (high-frequency band radar image); (e) two rebars in concrete slab (baseband radar image); (f) two rebars in concrete slab (high-frequency band radar image)

\section{Systematic Noise Reduction}

Reflection data acquired from an air-coupled GPR system are prone to contamination by various systematic noises, such as antenna direct coupling. These noise effects are the principal causes of GPR image background clutter. If their amplitudes are large, they can mask real rebar reflection signals. To remove the systematic noise, the averaging and subtraction approach is adopted. Because most of these systematic noises are deterministic and persist from waveform to waveform, time-domain averaging of the waveforms can remove the random and position-varying components to produce an estimate of the deterministic waveform signature. Then, by subtracting the averaged result from each waveform the deterministic noise can be greatly reduced. Figs. 11(a and b) show the resulting B-scan image and a trace waveform after systematic noise reduction. Comparing Figs. 10(b and c), it can be seen that the averaging and subtraction remove the background clutter and make the rebar signatures more pronounced.

\section{Radio-Frequency Interference Reduction}

The GPR data collection is also subjected to RF interference (RFI) in the test environment. Because RFI is transient and random, applying median filtering can often alleviate its effects. Median filtering examines a $3 \times 3$ window for image data resampling. It first sorts the nine data samples according to their amplitudes. Then, the median value point is selected to replace the center pixel in the resampling window. Fig. 11(c) shows the resulting image, and Fig. 11(d) is the signal waveform corresponding to the highlighted trace in Fig. 11(c).

\section{Target Area Detection}

After preprocessing, the GPR waveform data are arranged in a matrix $X$

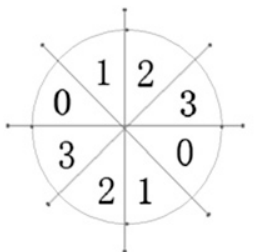

(a)

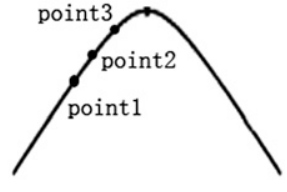

(b)
Fig. 15. Edge point detections: (a) four directions for gradient calculation; (b) three edge points on the hyperbola curve

$$
X=\left[\begin{array}{c}
X 11, X 12, \ldots, X 1 n \\
X 21, X 22, \ldots, X 2 n \\
\vdots \\
X m 1, X m 2, \ldots, X m n
\end{array}\right]
$$

where $n=$ number of traces and $m=$ sampling points on the trace. Vector $\mathbf{b}$ is the averaging vector

$$
\mathbf{b}=\left[b_{1} b_{2} \cdots b_{m}\right]^{T}
$$

where

$$
b_{i}=\frac{1}{n} \sum_{j=1}^{n} x_{i j}, \quad i=1,2, \ldots, m
$$

The normalized horizontal variance energy, $e_{i}$, and the normalized vertical variance energy, $e_{j}$, can be calculated, respectively, as

$$
e_{i}=\frac{1}{n} \sum_{j=1}^{n}\left(X_{i, j}-b_{i}\right)^{2}, \quad e_{j}=\frac{1}{m} \sum_{i=1}^{m}\left(X_{i, j}-b_{i}\right)^{2}
$$



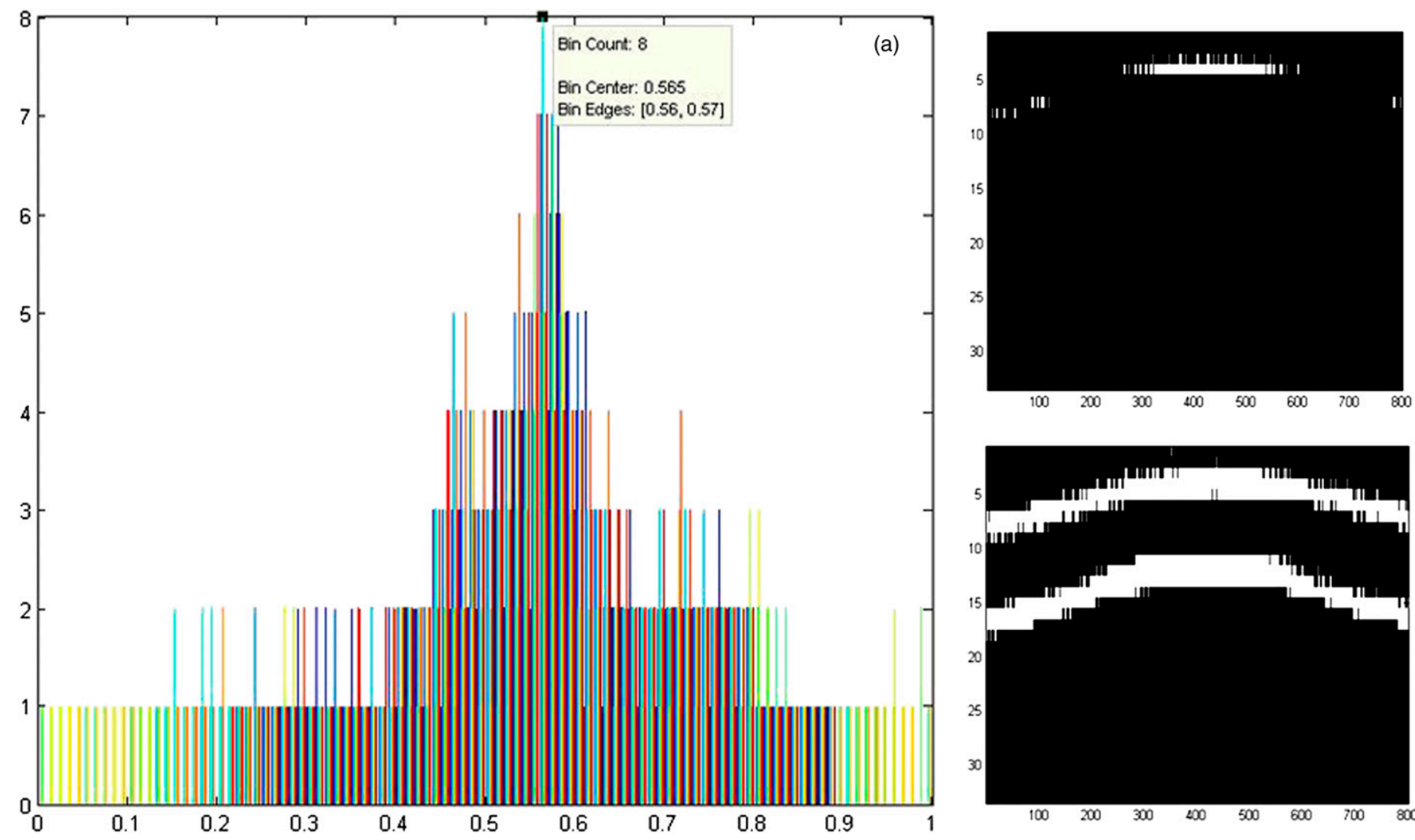

(b)

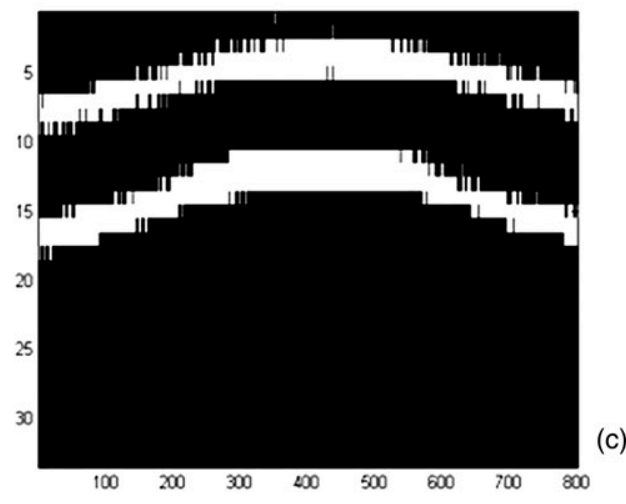

Fig. 16. Image segmentation with selected thresholds: (a) histogram of the image gradients; (b) image segmentation with $T_{1}$; (c) image segmentation with both $T_{1}$ and $T_{2}$

Table 1. Values of $T_{1}$ and $T_{2}$ in Various Images

\begin{tabular}{llcccc}
\hline Medium & $\begin{array}{c}\text { Band } \\
\text { frequency }\end{array}$ & $\begin{array}{c}\text { Standard } \\
\text { gradient }\end{array}$ & SD & $T_{1}$ & $T_{2}$ \\
\hline Air & Base & 0.565 & 0.289 & 0.854 & 0.565 \\
& Modulation & 0.525 & 0.300 & 0.825 & 0.525 \\
\multirow{2}{*}{ Sand } & Base & 0.425 & 0.200 & 0.625 & 0.425 \\
& Modulation & 0.435 & 0.145 & 0.580 & 0.435 \\
Concrete & Base & 0.445 & 0.255 & 0.700 & 0.445 \\
& Modulation & 0.455 & 0.265 & 0.720 & 0.455 \\
\hline
\end{tabular}

For the area with the buried rebar, both $e_{i}$ and $e_{j}$ values will experience abrupt changes because of the strong rebar reflection signal; therefore, an examination of the $e_{i}$ and $e_{j}$ patterns identifies the targeted detection area (Daniels 2004; van Kempen et al. 2000).

\section{Morphological Filtering}

After data preprocessing, there may still exist noise that could cause false target detection. In this step, morphological filtering is applied to improve the target area detection accuracy. The unique feature of morphology filtering is that it utilizes the geometric characteristics of the target to differentiate the target from the background. Because the geometric signature of the rebar is a hyperbola curve in the GPR B-scan image, the following structure element (SE) is thus utilized to perform morphological filtering:

$$
\mathrm{SE}=\left[\begin{array}{lll}
0 & 1 & 0 \\
1 & 1 & 0 \\
1 & 0 & 1
\end{array}\right]
$$

The resulting data after morphological filtering appear in Fig. 12. Figs. 13(a and c) plot the normalized horizontal and vertical variance energy curves before morphological filtering. Figs. 13(b and d) are the normalized horizontal and vertical variance energy curves after morphological filtering and low-pass filtering. Fig. 14 is the resulting GPR image. It is apparent that morphological filtering significantly cleans the energy maps and enhances the rebar hyperbolic traits.

\section{Hyperbola Fitting}

After identifying the target area, a hyperbola-fitting method extracts the signature curve for rebar depth calculations. It is important to first identify the edge points on the hyperbola curve to conduct curve fitting.

\section{Edge Point Detection}

There are many edge detection methods that have been investigated for GPR image processing. The present design adopts the Canny operator (Canny 1986). In comparison with other methods, the Canny operator has high detection accuracy in spotting continuous edge curves even when some real edge points may be marked by nearby strong interfering points. Such a scenario is typical for a highway GPR system that is operating under strong transient noise and interference. The basis of the Canny operator is that the edge under detection lies along a continuous curve with smooth transition gradients. Those points with abrupt gradient value changes can be discarded as noisy points. In addition, by following the derived curve direction, faint edge points with low gray-scale values can also be traced. Two gradient threshold values, $T_{1}$ and $T_{2}$, were utilized for the Canny operators, in which $T_{1}$ has a high value for filtering out the abrupt gradient changing points and $T_{2}$ has a low value for picking up faint edge points. 

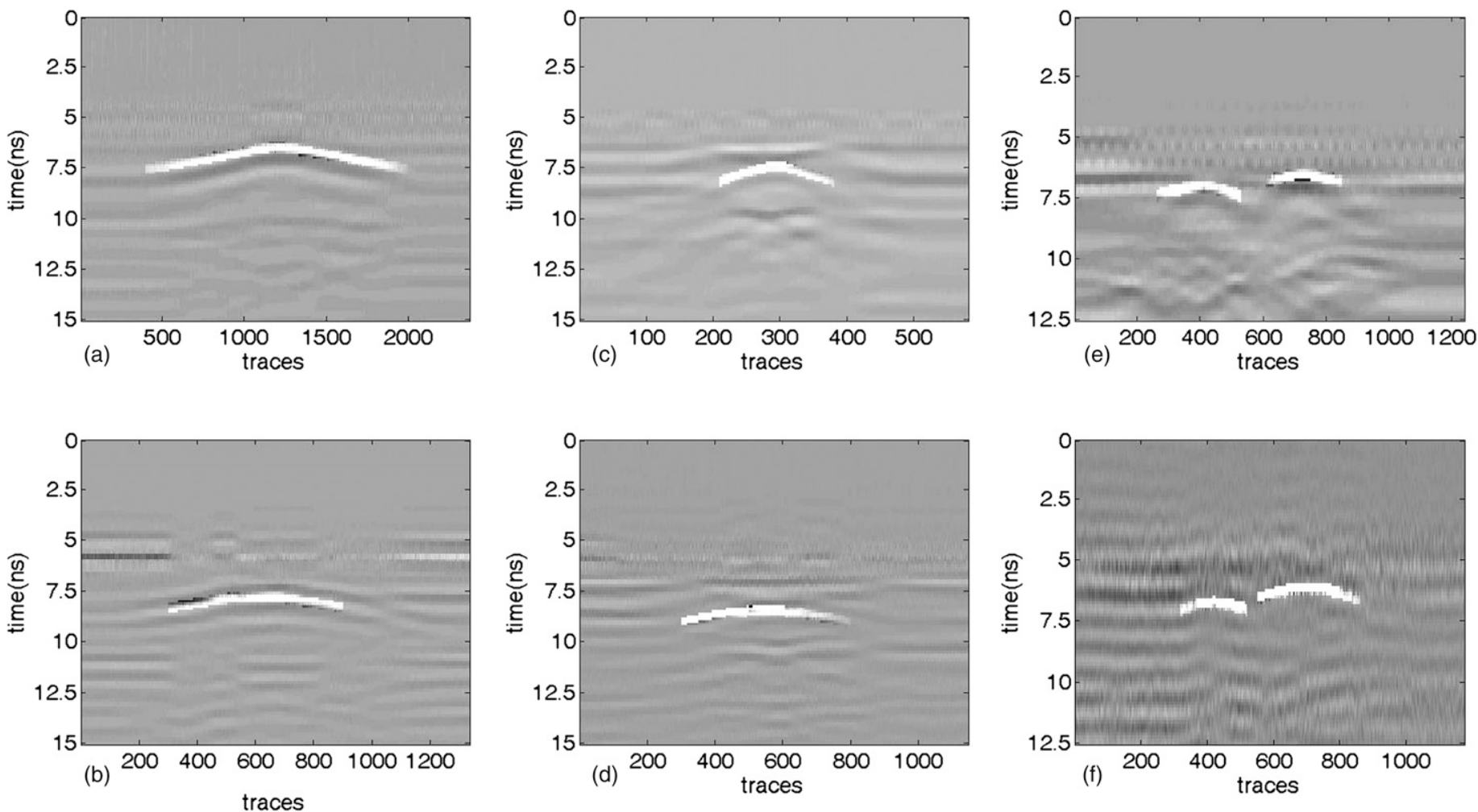

Fig. 17. Hyperbola fitting results: (a) one rebar in air (base-band radar image); (b) one rebar in air (high-frequency band radar image); (c) one rebar in sand (base-band radar image); (d) one rebar in sand (high-frequency band radar image); (e) two rebars in concrete slab (base-band radar image); (f) two rebars in concrete slab (high-frequency band radar image)
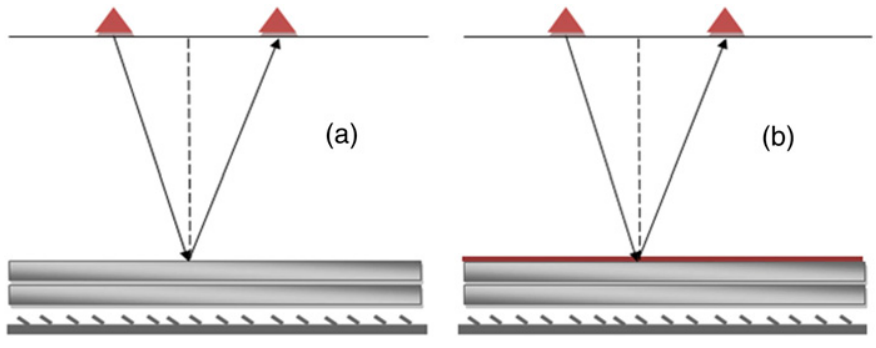

Fig. 18. Test setup for concrete dielectric constant estimation: (a) concrete under antenna; (b) concrete slab with a metal on the top surface

The implementation details of edge point detection are as follows:

1. In the gradient calculation, the magnitude and direction of the gradient of the image are calculated through the first-order partial derivative. For a hyperbolic curve, the gradient has four possible directions in the $3 \times 3$ neighborhood, which are shown in Fig.15(a), where the four sectors are numbered from 0 to 3.

2. In candidate edge point searching, a pixel is classified as an edge point if it meets the following conditions:

$a$. The value of this pixel is greater than the values of two adjacent pixels along the gradient direction, where the two adjacent points are in the same number sector in Fig. 15(a);

b. In Fig. 15(b), assume Points 1, 2, and 3 have been detected and are adjacent to each other. Using the $x$-axis as the reference direction, the direction angle of two points can be defined as the angle between the line segment connecting the two points and the $x$-axis. This allows
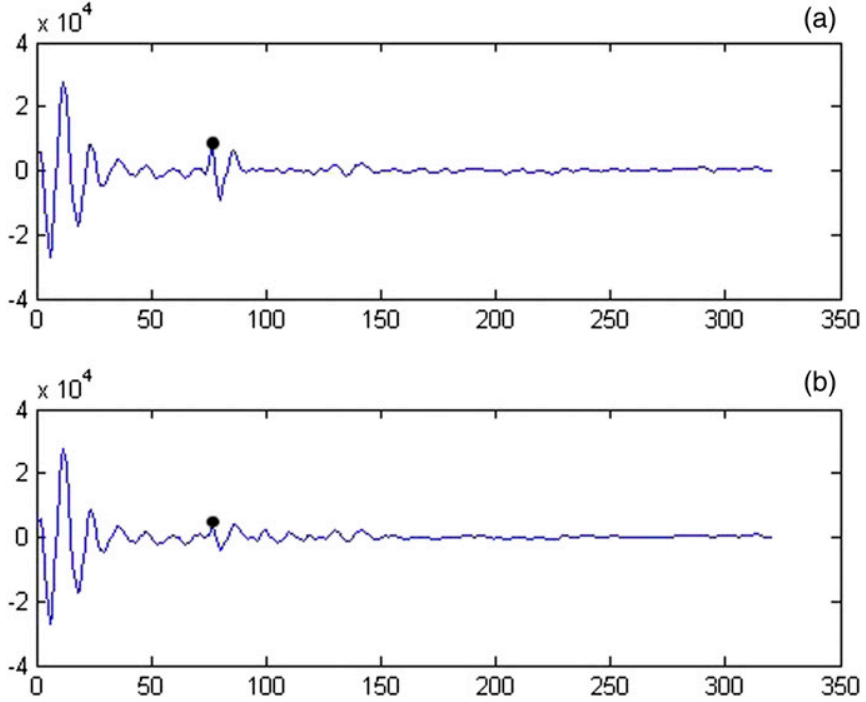

Fig. 19. Reflection signal waveforms from (a) a metal sheet and (b) a concrete slab

calculating the direction angle of Points 1 and 2 and Points 2 and 3. Because the edge points are the points on a smooth hyperbola curve, the difference between those two direction angles will be small. In the design, the angle difference is set to be less than $30^{\circ}$.

3. In edge detection and connection with Canny operator, applying the Canny method for edge point detection and connection 

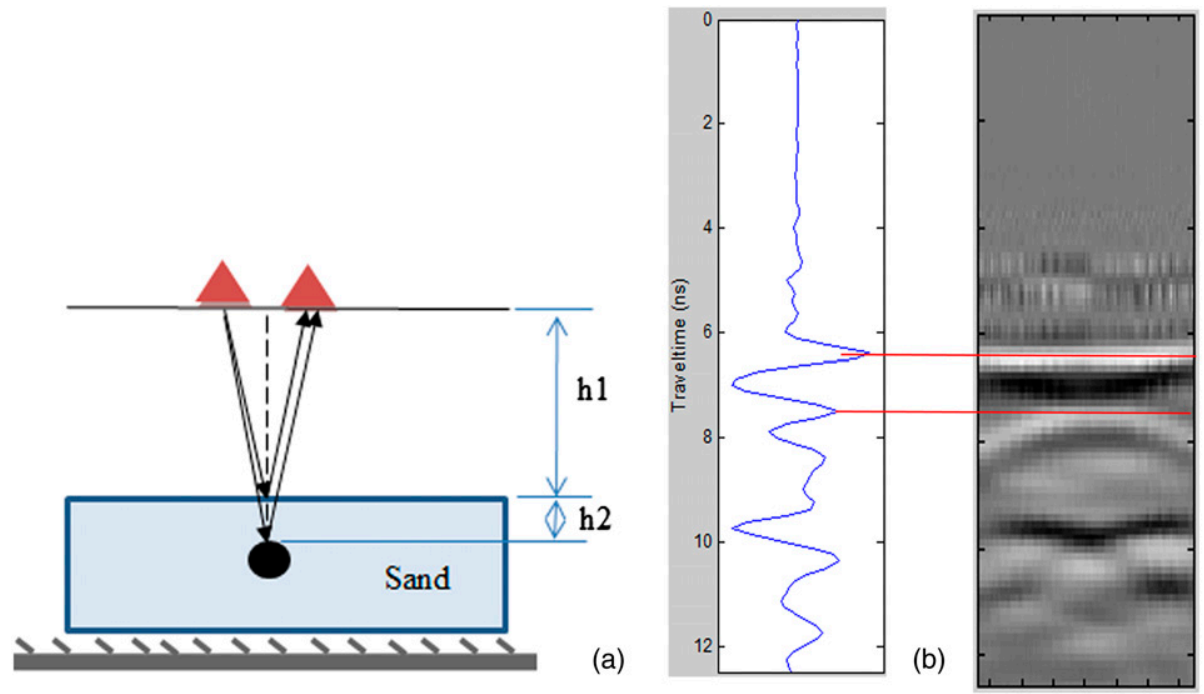

(c)

Fig. 20. Test diagram for estimating the dielectric constant of sand: (a) diagram of the test setup; (b) typical single signal trace; (c) assembled B-scan image

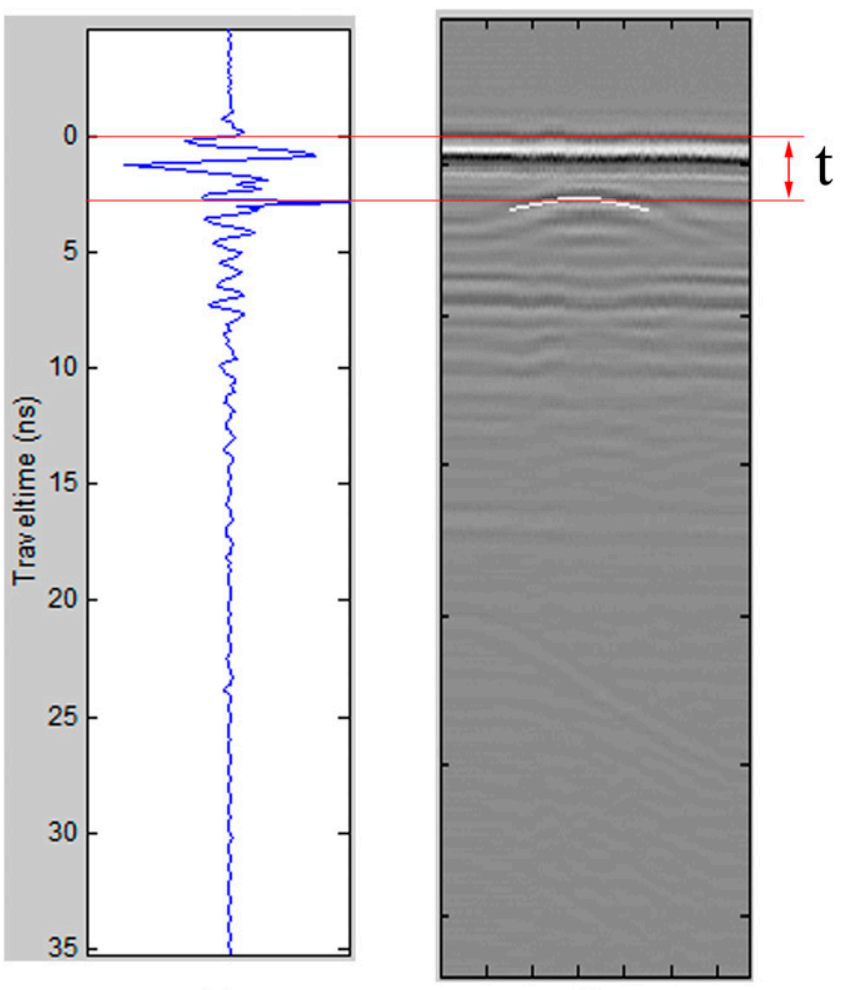

(a)

(b)

Fig. 21. Rebar scan data with wave travel time $t$ in a dielectric medium

requires selecting two threshold values, $T_{1}$ and $T_{2}$. Using the previously identified edge point candidates produces the gradient histogram in Fig. 16(a). Taking the stem value that has the largest frequency density, the $T_{2}$ value can be determined. In this design, it is 0.565 . The gradient standard deviation is 0.289 . The larger threshold $T_{1}$ value is thus set to 0.854 , which equals the sum of $T_{2}$ and one standard deviation. Fig. 16(b) plots the edge points detected by applying threshold $T_{1}$ only, while Fig. 16(c) plots the image after applying both $T_{1}$ and $T_{2}$. Figs. 17(a-f) show the effective detection of the edge points on the hyperbola curve. Performing the same processing steps, the $T_{1}$ and $T_{2}$ values for all radar images are obtained, which are summarized in Table 1.

\section{Hyperbola Curve Fitting}

Hyperbola fitting is applied after detecting edge points. Measuring the depth of the rebar curve in the test medium requires locating the hyperbolic apex point. This test development employs a linear fitting algorithm. The standard equation for a hyperbola is

$$
\left(y^{2} / a^{2}\right)-\left(x^{2} / b^{2}\right)=1
$$

where $a>0, b>0$, and $(x, y)=$ hyperbola coordinates; namely, the coordinates of the edge reflection of the target. Substituting $y^{2}$ with $u$ and $x^{2}$ with $v$ in Eq. (6) gives

$$
\left(u / a^{2}\right)-\left(v / b^{2}\right)=1
$$

Taking the coordinate values of all edge points detected, and applying linear hyperbola fitting, produces the values of parameters $a$ and $b$. For the rebar-in-air image, $a$ and $b$ are calculated to be 0.28 and 2.79, respectively. Fig. 17 shows the hyperbola fitting results for all six GPR images, with the pixels on the hyperbola curves being highlighted to improve visibility.

\section{Rebar Depth Estimation}

After locating the rebar hyperbola curve in the GPR image, it is possible to calculate its depth. This requires determining the EM wave propagation speed in the medium, which makes measuring the relative dielectric constant of the medium a necessity.

\section{Concrete Slab Dielectric Constant Measurement}

A simple yet effective GPR testing technique infers the dielectric constant of bulk materials from surface reflectivity measurements (Huston 2011). The reflection coefficient at the interface of two media is $R_{12}$, which equals the ratio of the electrical fields of the 
Table 2. Rebar Depth Estimation in Various Media

\begin{tabular}{|c|c|c|c|c|c|c|c|c|c|}
\hline Medium & $\begin{array}{c}\text { Rebar } \\
\text { number }\end{array}$ & $\begin{array}{c}\text { Band frequency } \\
(\mathrm{GHz})\end{array}$ & $\begin{array}{l}\text { Dielectric } \\
\text { constant }\end{array}$ & $\begin{array}{l}\text { Wave } \\
\text { speed }\end{array}$ & $\begin{array}{l}\text { Two-way travel } \\
\text { time (ns) }\end{array}$ & $\begin{array}{c}\text { Estimated } \\
\text { depth }(\mathrm{mm})\end{array}$ & $\begin{array}{c}\text { Actual } \\
\text { depth (mm) }\end{array}$ & $\begin{array}{l}\text { Error } \\
(\mathrm{mm})\end{array}$ & $\begin{array}{c}\text { Error } \\
(\%)\end{array}$ \\
\hline \multirow[t]{2}{*}{ Air } & \multirow[t]{2}{*}{1} & Base & 1 & $3 \times 10^{8}$ & 2.00 & 299.8 & 295 & 4.8 & 1.63 \\
\hline & & Modulation & 1 & $3 \times 10^{8}$ & 2.94 & 440.3 & 450 & -9.7 & 2.16 \\
\hline \multirow[t]{2}{*}{ Sand } & \multirow[t]{2}{*}{2} & Base & 3.5 & $1.60 \times 10^{8}$ & 1.17 & 93.6 & 100 & -6.4 & 6.4 \\
\hline & & Modulation & 3.5 & $1.60 \times 10^{8}$ & 1.345 & 107.6 & 10 & 7.6 & 7.6 \\
\hline \multirow[t]{4}{*}{ Concrete slab } & \multirow[t]{2}{*}{3} & Base & 4.1 & $1.48 \times 10^{8}$ & 1.58 & 116.6 & 107.6 & 9 & 8.4 \\
\hline & & Modulation & 4.1 & $1.48 \times 10^{8}$ & 1.375 & 101.8 & 107.6 & -5.8 & 5.3 \\
\hline & \multirow[t]{2}{*}{4} & Base & 4.1 & $1.48 \times 10^{8}$ & 1.25 & 92.5 & 98.6 & -6.1 & 6.18 \\
\hline & & Modulation & 4.1 & $1.48 \times 10^{8}$ & 1.195 & 88.5 & 98 & -10.1 & 10.2 \\
\hline
\end{tabular}

incident wave and the reflection wave. The $R_{12}$ value depends on the dielectric constants $\varepsilon_{1}$ and $\varepsilon_{2}$ of the two media

$$
R_{12}=\frac{\sqrt{\varepsilon_{1}}-\sqrt{\varepsilon_{2}}}{\sqrt{\varepsilon_{1}}+\sqrt{\varepsilon_{2}}}
$$

A test method, illustrated in Fig. 18, was designed to estimate the dielectric constant of the concrete slab with the assistance of a thin metal sheet. Because the signal amplitude is proportional to the electric field, by measuring the reflection pulse amplitude, $A_{c}$, from the concrete surface and the reflection pulse amplitude, $A_{\mathrm{pl}}$, from the metal plate, the reflection ratio can be calculated as $R_{12}=-A_{c} / A_{\mathrm{pl}}$. Thus, the dielectric constant of the concrete slab equals

$$
\varepsilon_{c}=\left[\frac{1+\left(A_{c} / A_{\mathrm{pl}}\right)}{1-\left(A_{c} / A_{\mathrm{pl}}\right)}\right]^{2}
$$

In Fig. 19, the quantized reflection signal amplitudes $A_{c}$ and $A_{\mathrm{pl}}$ equal 7,831 and 23,091, respectively. Using Eq. (9), the relative dielectric constant $\varepsilon_{c}$ of the concrete is estimated to be 4.1 .

\section{Dry Sand Dielectric Constant Measurement}

The size of the sandbox was 560 (width) $\times 270$ (length) $\times 240 \mathrm{~mm}$ (height), which is relatively small for dielectric measurements using the aforementioned bulk material surface reflectivity characterization method. To deduce the dielectric constant of the sand, $\varepsilon_{s}$, it was decided to measure the EM wave propagation speed in the sand directly. As is well known, for a medium with a dielectric constant $\varepsilon$, the speed of waves propagating in this medium and the round-trip traveling time are

$$
\begin{aligned}
& V=C / \sqrt{\varepsilon} \\
& t=h /(2 \cdot V)
\end{aligned}
$$

In the test shown in Fig. 19(a), a rebar was buried inside the sandbox with a previously known depth $(80 \mathrm{~mm})$ in the setup. The dielectric constant $\varepsilon_{s}$ can thus be obtained as

$$
\varepsilon_{s}=\left(C \cdot \frac{t_{2}-t_{1}}{2 \cdot h_{2}}\right)^{2}
$$

where $t_{1}=$ two-way traveling time between the top surface of the sand and the antennas of the EM wave, $t_{2}=$ two-way traveling time from the rebar in the sand and antennas, and $h_{2}=$ rebar depth in the sand $(80 \mathrm{~mm})$. From the scan data in Figs. 20(b and c), the $t_{2}$ and $t_{1}$ offset value is measured as $1 \times 10^{-9} \mathrm{~s}$. Applying Eq. (12), the relative dielectric constant of the sand is calculated to be 3.51 .

\section{Rebar Depth Estimation}

After measuring the relative dielectric constants, the wave propagation speed in the medium can be calculated using Eq. (10). The rebar depth in the dielectric medium can be determined using the hyperbolic apex coordinates in the radar image. For instance, in Fig. 21, the two-way EM wave traveling time $t$ is measured to be $1.69 \mathrm{~ns}$. The rebar depth can be calculated with Eqs. (10) and (11). Table 2 lists the rebar cover estimation results from the various test setups.

\section{Conclusions}

A new dual-band dual-channel air-launched UWB GPR was developed to facilitate highway and bridge inspection. In comparison with other systems in the literature, the performance of this GPR design is greatly improved using new design techniques. From a hardware design perspective, a UWB pulse generator is developed with novel yet simple impedance-matching and differentiator circuit technology, which achieves high pulse amplitude and low-level ringing. By using the 8-GSPS real-time data acquisition unit to accelerate reflection signal sampling, the GPR survey speed is significantly improved, which makes it applicable to highway and bridge inspection at regular driving speeds. Moreover, multithreaded parallel operations are implemented to speed up the large volume of data transmission and storage generated by the highspeed digitizer. From a signal-processing perspective, customized signal-processing methods, including data preprocessing, target area detection, and hyperbola fitting, have been developed to reduce systematic noise and RF interference, and to leverage subject detection accuracy. The experimental results show that this GRP can achieve high detection accuracy with measurement errors of less than $10 \%$. Future research directions are to further improve the GPR performance and to characterize the rebar dimensional size and corrosion conditions.

\section{Acknowledgments}

This research was supported by U.S. Department of Transportation Grant No. DTOS5908G00102 and U.S. National Institutes of Standards and Technology Cooperative Agreement No. 70NANB9H9012, with Northeastern University and Ming Wang as the principal investigator.

\section{References}

Al-Qadi, I. L., Lahouar, S., and Jiang, K. (2004). "Measuring rebar coverdepth in rigid pavements using GPR." Structural Materials Technology VI NDT Conf., S. Alampalli and G. Washer, eds., American Society of Nondestructive Testing, Columbus, $\mathrm{OH}$. 
Borgioli, G., Capineri, L., Falorni, P., Mattucci, S., and Windsor, C. G. (2008). "The detection of buried pipes from time-of-flight radar data." IEEE Trans. Geosci. Remote Sens., 46(8), 2254-2266.

Canny, J. (1986). "A computational approach to edge detection." IEEE Trans. Pattern Anal. Mach. Intell., 8(6), 679-698.

Chang, C. W., Lin, C. H., and Lien, H. S. (2009). "Measurement radius of reinforcing steel bar in concrete using digital image GPR." Constr. Build. Mater., 23(2), 1057-1063.

Daniels, D. J. (2004). Ground penetrating radar, 2nd Ed., Institute of Electrical Engineers, London.

Dérobert, X., Iaquinta, J., Klysz, G., and Balayssac, J.-P. (2008). "Use of capacitive and GPR techniques for the non-destructive evaluation of cover concrete." NDT \& E Int., 41(1), 44-52.

Federal Communications Commission (FCC). (2002). "Revision of Part 15 of the Commision's rules regarding ultra-wideband transmission systems." FCC 02-48, Washington, DC.

Gan, L., Zhou, L., You, X. (2011). "The time-frequency analysis of equivalenttime sampling GPR signal based on HHT." Int. Conf. on Computational and Information Sciences (ICCIS), IEEE, Piscataway, NJ, 929-932.

Halabe, U. B., Maser, K. R., and Kausel, E. A. (1995). "Condition assessment of reinforced concrete structures using electromagnetic waves." ACI Mater. J., 92(5), 511-523.

Huston, D. (2011). Structural sensing health monitoring and performance evaluation, CRC, Boca Raton, FL, 117.

Huston, D. R., Hu, J. Q., Maser, K., Weedon, W., and Adam, C. (2000). "GIMA ground penetrating radar system for infrastructure health monitoring." J. Appl. Geophys., 43(2-4), 139-146.

Johansson, E. M., and Mast, J. E. (1994). "Three-dimensional ground penetrating radar imaging using synthetic aperture time-domain focusing." SPIE Proc., S. Udpa and H. Han, eds., 2275, 205-524.

Jol, H. M. (2009). Ground penetrating radar theory and applications, Elsevier, Amsterdam, Netherlands.

Kim, Y. J., Jofre, L., De Flaviis, F., and Feng, M. Q. (2004). "Microwave subsurface imaging technology for damage detection." J. Eng. Mech., 130(7), 858-866.
Olhoeft, G. R. (2000). "Maximizing the information return from ground penetrating radar." J. Appl. Geophys., 43(2-4), 175187.

Pasolli, E., Melgani, F., and Donelli, M. (2009). "Automatic analysis of GPR images: A pattern-recognition approach." IEEE Trans. Geosci. Remote Sens., 47(7), 2206-2217.

Roqueta, G., Jofre, L., and Feng, M. Q. (2012). "Analysis of the electromagnetic signature of reinforced concrete structures for nondestructive evaluation of corrosion damage." IEEE Trans. Instrum. Meas., 61(4), 1090-1098.

Shaw, M. R., Molyneaux, T. C. K., Millard, S. G., Taylor, T. J., and Bungey, J. H. (2003). "Assessing bar size of steel reinforcement in concrete using ground penetrating radar and neural networks." Insight NonDestr. Test. Cond. Monit., 45(12), 813-816.

Shihab, S., and Al-Nuaimy, W. (2005). "Radius estimation for cylindrical objects detected by ground penetrating radar." Subsurf. Sens. Technol. Applic., 6(2), 151-166.

Uddin, W. (2006). "Ground penetrating radar study_Phase I: Technology review and evaluation." FHWA/MS-DOT-RD-06-182, Federal Highway Administration, Washington, DC.

van Kempen, L., Sahli, H., Brooks, J., and Cornelis, J. (2000). "New results on clutter reduction and parameter estimation for landmine detection using GPR." Proc., 8th Int. Conf. on Ground Penetrating Radar, Society of Photo-Optical Instrumentation Engineers, Bellingham, WA, 872-879.

Wang, Z. W., Zhou, M., Slabaugh, G. G., Zhai, J., and Fang, T. (2011). "Automatic detection of bridge deck condition from ground penetrating radar images." IEEE Trans. Autom. Sci. Eng., 8(3), 633640.

Xia, T., Venkatachalam, A. S., and Huston, D. (2012). "A high performance low ringing ultra-wideband monocycle pulse generator." IEEE Trans. Instrum. Meas., 61(1), 261-266.

Yang, J. (2011). "The research of detection of the underground pipe's diameter based on GPR." M.S. thesis, China Univ. of Mining and Technology, Beijing, 31-33. 